\title{
Kondo and Fano Effect in Side Attached Double Quantum-Dot Molecule
}

\author{
Gustavo A. Lara*, Pedro A. Orellana*, Julio M. Yáñez*, and Enrique V. Anda ${ }^{\dagger}$ \\ * Departamento de Física, Universidad de Antofagasta, Casilla 170, Antofagasta, Chile \\ * Departamento de Física, Universidad Católica del Norte, Casilla 1280, Antofagasta, Chile \\ ${ }^{\dagger}$ Departamento de Física, P. U. Católica do Rio de Janeiro, C.P. 38071-970, Rio de Janeiro, RJ, Brazil
}

\section{Received on 8 December, 2005}

\begin{abstract}
Electron tunneling through a double quantum dot molecule side attached to a quantum wire, in the Kondo regime, is studied. The mean-field finite- $U$ slave-boson formalism is used to obtain the solution of the problem. We investigate the many body molecular Kondo state and its interplay with the inter-dot antiferromagnetic correlation as a function of the parameters of the system.
\end{abstract}

Keywords: Quantum dots; Kondo effect; Fano effect

\section{INTRODUCTION}

The Kondo effect in quantum dots (QDs) has been extensively studied in the last years [1]. The QDs allow to study systematically the quantum-coherence many-body Kondo state, due to the possibility of continuous tuning the relevant parameters governing the properties of this state, in equilibrium and non-equilibrium situations. Recently Kondo effect has been studied in double quantum dot molecule in series $[2,3]$. This system allows the study of the many body molecular Kondo states in equilibrium and non-equilibrium situation. The type of coupling between the QDs determines the character of the electronic states and the transport properties of the artificial molecule. In the tunneling regime, the electronics states are extended across the entire system and form a coherent state based on the bonding or anti-bonding levels of the QDs.

An alternative configuration consists of a side-coupled QDs attached to a perfect quantum wire (QW). This structure is reminiscent of T-shaped quantum wave guides known as electron stub tuners [4]. In this case, the QDs act as scattering centers in close analogy with the traditional Kondo effect [5].

Recent electron transport experiments showed that Kondo and Fano resonances occurs simultaneously [6]. Multiple scattering of traveling electronic waves on a localized magnetic state are crucial for a formation of both resonances. The condition for the Fano resonance [7] is the existence of two scattering channels: a discrete level and a broad continuum band $[8,9]$.

In this work we study the transport properties of a double quantum dot molecule side attached to a quantum wire in the Kondo regimen. We use the finite- $U$ slave boson meanfield approach which was initially developed by Kotliar and Ruckenstein [10] and used later by Bing Dong and X. L. Lei to study the transport through coupled double quantum dots connected to leads [11], which enforces the correspondence between the impurity fermions and the auxiliary fermions to a mean-field level to release the $U=\infty$ restriction. In double quantum dot molecules this approach allows one to treat both dot-lead coupling and the interdot tunneling nonperturbatively at an arbitrary strengths of the Coulomb correlation $U$, and thus an antiferromagnetic exchange coupling parameter ap- pears naturally [11]. We investigate the many body molecular Kondo ground state and the competing singlet ground state that results from the anti-ferromagnetic correlation between the dots, as a function of the parameters of the system. In the Kondo regime, each dot results to have its own Kondo temperature as a consequence of their different connections to the conduction electrons.

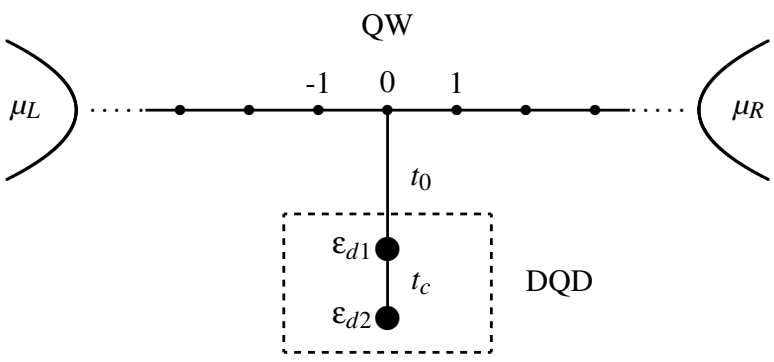

FIG. 1: Scheme of double quantum dot (DQD) attached to a lead (perfect quantum wire $(\mathrm{QW})$ ). The $\mathrm{QW}$ is coupled to the left $(L)$ and right $(R)$.

\section{MODEL}

Let us consider a double quantum dot (DQD) side coupled to a perfect quantum wire (QW) (see Fig. 1). We adopt the two-fold Anderson Hamiltonian. Each dot has a single level $\varepsilon_{d i}$ (with $i=1,2$ ), intra-dot Coulomb repulsion $U$, is coupled to each other with inter-dot tunneling coupling $t_{c}$. The side attached double quantum-dot is coupled to the $\mathrm{QW}$ with coupling $t_{0}$. The QW has a site energy $\varepsilon_{i, \sigma}$ and a hopping parameter $t$.

In analogy with the infinite- $U$ slave boson approach [12] the Hilbert space is enlarged at each site, to contain in addition to the original fermions a set of four bosons [10] represented by the creation (annihilation) operators $e_{i}^{\dagger}\left(e_{i}\right), p_{i, \sigma}^{\dagger}\left(p_{i, \sigma}\right)$, and $d_{i}^{\dagger}\left(d_{i}\right)$ for the $i$-th dot, which act, respectively, as projectors onto empty, single occupied (with spin up and down) and doubly occupied electron states. The Hamiltonian is written as, 


$$
\begin{aligned}
H= & -t \sum_{i, \sigma}\left(c_{i, \sigma}^{\dagger} c_{i+1, \sigma}+\text { H.c. }\right)-\sum_{\sigma} \tilde{t}_{0, \sigma}\left(c_{0, \sigma}^{\dagger} f_{1, \sigma}+\text { H.c. }\right) \\
& +\sum_{i=1}^{2} \sum_{\sigma} \tilde{\varepsilon}_{d i, \sigma} f_{i, \sigma}^{\dagger} f_{i, \sigma}-\sum_{\sigma} \tilde{t}_{c, \sigma}\left(f_{1, \sigma}^{\dagger} f_{2, \sigma}+\text { H.c. }\right) \\
& +\sum_{i=1}^{2}\left\{U d_{i}^{2}+\lambda_{i}^{(1)}\left(p_{i, \uparrow}^{2}+p_{i, \downarrow}^{2}+e_{i}^{2}+d_{i}^{2}-1\right)-\sum_{\sigma} \lambda_{i, \sigma}^{(2)}\left(p_{i, \sigma}^{2}+d_{i}^{2}\right)\right\},
\end{aligned}
$$

where $c_{i, \sigma}^{\dagger}\left(c_{i, \sigma}\right)$ is the creation (annihilation) operator of an electron with spin $\sigma$ in the $i$-th site on quantum wire; $f_{i, \sigma}^{\dagger}\left(f_{i, \sigma}\right)$ is the creation (annihilation) operator of an electron with spin $\sigma$ in the i-th QD, $\tilde{\varepsilon}_{d i, \sigma}=\varepsilon_{d i}+\lambda_{i, \sigma}^{2}, \tilde{t}_{0, \sigma}=t_{0}\left\langle\tilde{Z}_{1, \sigma}\right\rangle$, and $\tilde{t}_{c, \sigma}=$
$t_{c}\left\langle\tilde{Z}_{1, \sigma} \tilde{Z}_{2, \sigma}\right\rangle$, and we set $\varepsilon_{i, \sigma}=0$ in the QW. The operator $\tilde{Z}_{i, \sigma}$ is chosen to reproduce the correct $U \rightarrow 0$ limit in the mean field approximation,

$$
\tilde{Z}_{i, \sigma}=\left(1-d_{i}^{\dagger} d_{i}-p_{i, \sigma}^{\dagger} p_{i, \sigma}\right)^{-1 / 2}\left(e_{i}^{\dagger} p_{i, \sigma}+p_{i,-\sigma}^{\dagger} d_{i}\right)\left(1-e_{i}^{\dagger} e_{i}-p_{i,-\sigma}^{\dagger} p_{i,-\sigma}\right)^{-1 / 2}
$$

The constraint, i.e., the completeness relation $\sum_{\sigma} p_{i, \sigma}^{\dagger} p_{i, \sigma}+$ $b_{i}^{\dagger} b_{i}+d_{i}^{\dagger} d_{i}=1$ and the condition for the correspondence between fermions and bosons $f_{i, \sigma}^{\dagger} f_{i, \sigma}=p_{i, \sigma}^{\dagger} p_{i, \sigma}+d_{i}^{\dagger} d_{i}$, have been incorporated with Lagrange multipliers $\lambda_{i}^{(1)}$ and $\lambda_{i, \sigma}^{(2)}$ into the Hamiltonian. We use the mean-field approximation in which all the boson operators are replaced by their expectation value. As we work at zero temperature these expectation values and the Lagrange multipliers are then determined by minimization of the energy with respect to these variables.

We resolve the problem using the method developed in Ref[3]. Resolving the equations of motion, we find the transmission amplitude,

$$
\tau=\frac{\left(\omega-\tilde{\varepsilon}_{-}\right)\left(\omega-\tilde{\varepsilon}_{+}\right)}{\left(\omega-\tilde{\varepsilon}_{-}\right)\left(\omega-\tilde{\varepsilon}_{+}\right)+i\left(\omega-\tilde{\varepsilon}_{d 2}\right) \tilde{\Gamma}},
$$

where the renormalized bonding and anti-bonding energies $\left(\tilde{\varepsilon}_{ \pm}\right)$are defined by $\tilde{\varepsilon}_{ \pm}=\left(\tilde{\varepsilon}_{d 1}+\tilde{\varepsilon}_{d 2}\right) / 2 \pm$ $\sqrt{\left.\left(\tilde{\varepsilon}_{d 1}+\tilde{\varepsilon}_{d 2}\right) / 2\right)^{2}+\tilde{t}_{c}^{2}}$ and $\tilde{\Gamma}=\pi \tilde{t}_{0}^{2} \rho_{0}(\omega)$ is the renormalized coupling between the double quantum-dot and the leads of density of states $\rho_{0}(\omega)$. The transmission amplitude can be written in the polar form as, $\tau=|\tau| e^{i \phi}$, where $\phi$ is the transmission phase and it is given by,

$$
\phi=\tan ^{-1}\left(\frac{\mathfrak{I} \tau}{\mathfrak{R} \tau}\right)=\tan ^{-1}\left[\frac{-\left(\omega-\tilde{\varepsilon}_{d 2}\right) \tilde{\Gamma}}{\left(\omega-\tilde{\varepsilon}_{-}\right)\left(\omega-\tilde{\varepsilon}_{+}\right)}\right]
$$

The transmission probability is $T=|\tau|^{2}$,

$$
T(\omega)=\frac{\left[\left(\omega-\tilde{\varepsilon}_{-}\right)\left(\omega-\tilde{\varepsilon}_{+}\right)\right]^{2}}{\left[\left(\omega-\tilde{\varepsilon}_{-}\right)\left(\omega-\tilde{\varepsilon}_{+}\right)\right]^{2}+\left[\left(\omega-\tilde{\varepsilon}_{d 2}\right) \tilde{\Gamma}\right]^{2}} .
$$

Moreover we can obtain the density of states of the quantum dots system,

$$
\rho(\omega)=\frac{1}{\pi} \frac{\tilde{\Gamma}\left(\omega-\tilde{\varepsilon}_{d 2}\right)^{2}}{\left[\left(\omega-\tilde{\varepsilon}_{-}\right)\left(\omega-\tilde{\varepsilon}_{+}\right)\right]^{2}+\left[\left(\omega-\tilde{\varepsilon}_{d 2}\right) \tilde{\Gamma}\right]^{2}}+\frac{1}{\pi} \frac{\tilde{\Gamma} \tilde{t}_{c}^{2}}{\left[\left(\omega-\tilde{\varepsilon}_{-}\right)\left(\omega-\tilde{\varepsilon}_{+}\right)\right]^{2}+\left[\left(\omega-\tilde{\varepsilon}_{d 2}\right) \tilde{\Gamma}\right]^{2}}
$$

\section{RESULTS}

We take typical values for the parameters that define the system, $\mu=0, t=25 \Gamma, t_{0}=7.07 \Gamma$, where $\Gamma=\pi t_{0}^{2} \rho_{0}(0)$.

We consider first the situation where the two dots local state energies are varied simultaneously by a gate voltage $V_{g}$, i.e. $\varepsilon_{d 1}=\varepsilon_{d 2}=V_{g}$.

The transmission probability $T$ is displayed in Figure 2 for various values of $U$ and two values of $t_{c}$. The transmission probability always reaches zero at $\omega=\tilde{\varepsilon}_{-}$and $\tilde{\varepsilon}_{+}$and a unitary value at $\omega=\tilde{\varepsilon}_{d 2}$. The figure allows to study the interplay 


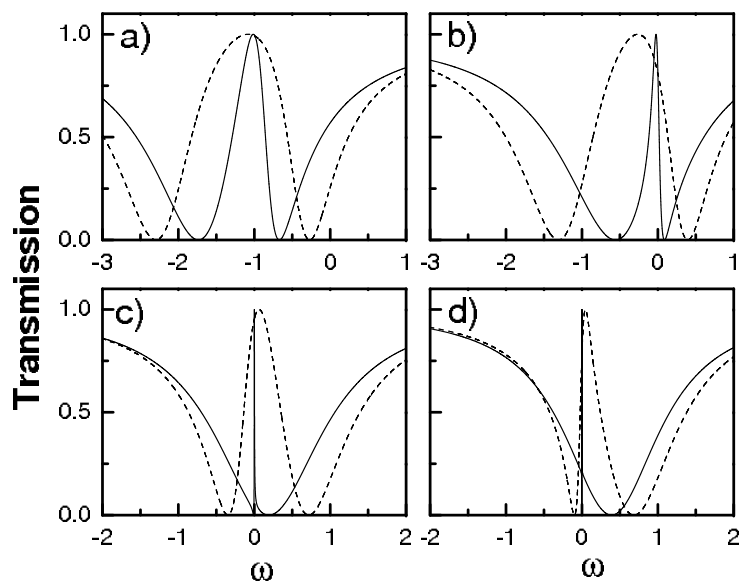

FIG. 2: Transmission spectrum for $V_{1 g}=V_{2 g}=V_{g}=-3 \Gamma, t_{c}=0.5 \Gamma$ (solid line) and $t_{c}=\Gamma$ (dashed line), for various values of the on site energy $U$, a) $U=2 \Gamma$, b) $U=4 \Gamma$, c) $U=8 \Gamma$ and d) $U=16 \Gamma$.

between the Kondo effect and the inter-dot anti-ferromagnetic correlation. Increasing $U$, a sharp feature develops close to the Fermi energy $(\omega=0)$, indicating the appearance of a Kondo resonance. We can see that this process is more rapidly defined for the case where $t_{c}=0.5 \Gamma$ than for $t_{c}=\Gamma$. This behavior is due to the anti-ferromagnetic interaction between the spins of the dots, proportional to $t_{c}^{2} / U$ [11], that destroys the Kondo effect when it is greater than the Kondo temperature creating a singlet ground state for the dot molecule.

For $U$ sufficiently large the transmission can be written approximately as the superposition of two Fano-Kondo line shapes, one of them has zero $q$ factor,

$$
T(\omega) \approx \frac{\left(\varepsilon^{\prime}+q^{\prime}\right)^{2}}{\left(\varepsilon^{\prime}\right)^{2}+1}+\frac{\left(\varepsilon^{\prime \prime}+q^{\prime \prime}\right)^{2}}{\left(\varepsilon^{\prime \prime}\right)^{2}+1},
$$

where $\varepsilon^{\prime}=\left(\omega-\tilde{\varepsilon}_{d 2}\right) / \tilde{\Delta}, q^{\prime}=\left(\tilde{\varepsilon}_{d 2}-\tilde{\varepsilon}_{-}\right) / \tilde{\Delta}, \varepsilon^{\prime \prime}=\left(\omega-\tilde{\varepsilon}_{+}\right) / \Gamma$ and $q^{\prime \prime}=0$, with $\tilde{\Delta}=\tilde{t}_{c}^{2} / \tilde{\Gamma}$.

The DOS of the quantum-dot molecule give us a better understanding of the transport properties of the system. The DOS is shown in Fig. 3 for various values of $U$, for the case $t_{c}=0.5$, where the Kondo spin correlation is dominant over the anti-ferromagnetic one. We can observe the superposition of a narrow and a broad Kondo peak with widths $\tilde{\Delta}$ and $\tilde{\Gamma}$ respectively. In fact, the DOS can be written as the superposition of two Lorentzian,

$$
\rho(\omega) \approx \frac{1}{\pi} \frac{\tilde{\Gamma}}{\left(\omega-\tilde{\varepsilon}_{+}\right)^{2}+\tilde{\Gamma}^{2}}+\frac{1}{\pi} \frac{\tilde{\Delta}}{\left(\omega-\varepsilon_{d 2}\right)^{2}+\tilde{\Delta}^{2}} .
$$

As it can be seen from the figure there is no Kondo peak for $U=2 \Gamma$, as it should because in this case the QDs are with two electrons each.

In order to confirm the destructive effect of antiferromagnetism on the Kondo regime, we display in the inset of the figure the DOS of the DQD for the same parameters as before but with $t_{c}=\Gamma$. The DOS shows two peaks at the bonding and anti-bonding energies with no Kondo peak, showing that for this value of $t_{c}$ anti-ferromagnetism is dominant.

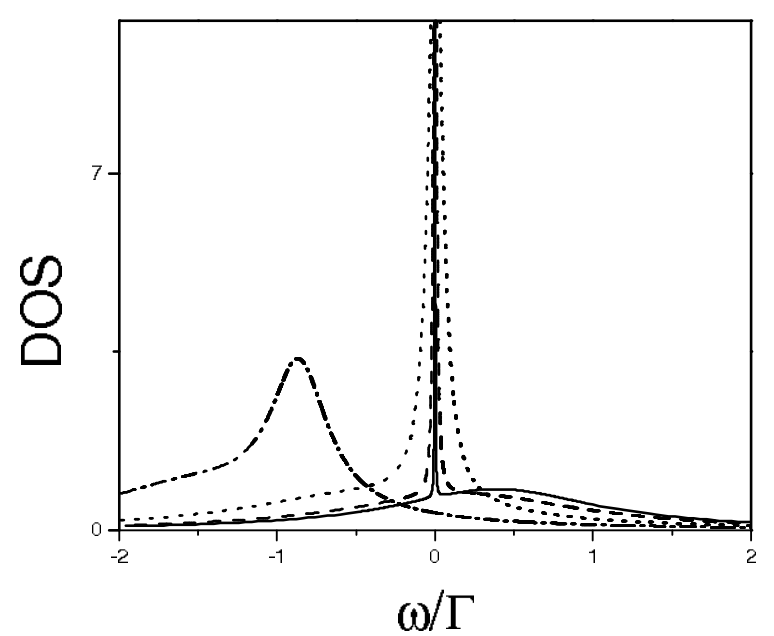

FIG. 3: DOS for $t_{c}=0.5 \Gamma, V_{1 g}=V_{2 g}=-3 \Gamma$, for various values of the on site energy, $U=16 \Gamma$ (solid line), $U=8 \Gamma$ (dashed line), $U=4 \Gamma$ (doted line) and $U=2 \Gamma$ (dash-dot line).

On the other hand, we can evaluate the transmission phase at the Fermi energy,

$$
\phi(0)=\tan ^{-1}\left[\frac{\tilde{\varepsilon}_{d 2} \tilde{\Gamma}}{\tilde{\varepsilon}_{-} \tilde{\varepsilon}_{+}}\right] .
$$

Figure 4 displays the behavior of the transmission phase evaluated at the Fermi energy as a function of the gate voltage, for the on-site energy $U=8 \Gamma$. For large negative $V_{g}$ the energies $\tilde{\varepsilon}_{-}, \tilde{\varepsilon}_{+}$and $\tilde{\varepsilon}_{d 2}$ are negative and $\phi$ is negative and tends to zero. When $\tilde{\varepsilon}_{+}$goes to zero, $\phi$ tends to $-\pi / 2$. However, when $\tilde{\varepsilon}_{+}$becomes positive, $\phi$ jumps to $+\pi / 2$. For $V_{g}=U / 2$, $\tilde{\varepsilon}_{d 2}$ crosses the Fermi energy and the phase vanishes. At this gate voltage the transmission amplitude evaluated at the Fermi energy is a real number. Increasing $V_{g}$ when $\tilde{\varepsilon}_{-}$approaches to the Fermi energy, $\phi$ tends to $-\pi / 2$. At this gate voltage the phase suffers an abrupt change and jumps to $+\pi / 2$. For large positive values of $V_{g}$, the renormalized energies $\tilde{\varepsilon}_{-}, \tilde{\varepsilon}_{+}$and $\tilde{\varepsilon}_{d 2}$ are all positive and $\phi$ is positive and tends to zero.

\section{CONCLUSIONS}

We have studied the transport through a side-coupled double quantum-dot molecule using the finite- $U$ slave boson mean field approach. We have found that the transmission spectrum shows a structure with two Fano anti-resonances localized at the bonding and anti-bonding renormalized energies of the quantum-dot molecule, and one resonance at the renormalized site energy of the outside quantum-dot. The DOS shows that when the Kondo correlations are dominant both dots are at the Kondo regime each one with its own Kondo temperature. Increasing the inter-dot interaction, the anti-ferromagnetic correlation becomes dominant destroying 


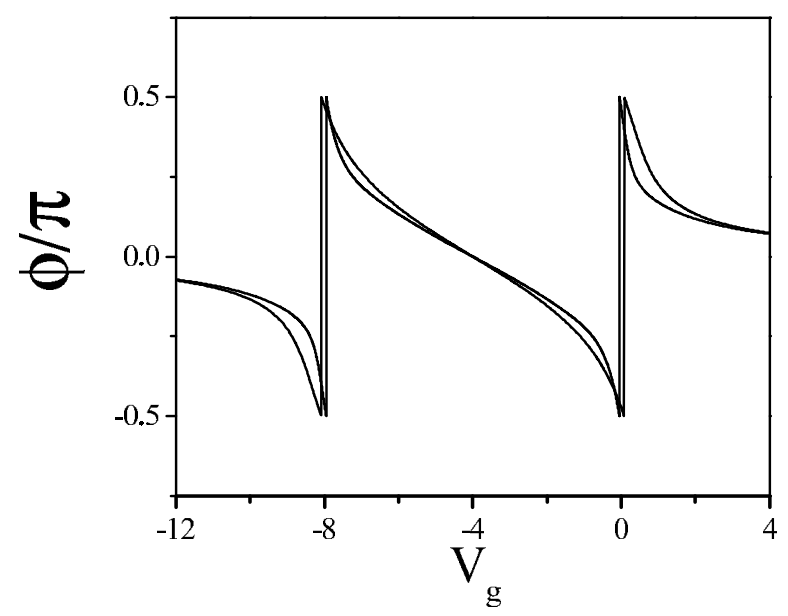

FIG. 4: Transmission phase $\phi$ vs $V_{g}$ for $t_{c}=0.5 \Gamma$ (solid line) and $t_{c}=\Gamma$, for on site energy, $U=8 \Gamma$. the Kondo effect and the physics associated with it. These phenomena have been analyzed as a function of the relevant parameters of the system.

\section{Acknowledgments}

G.A.L. and P.A.O. would like to thank financial support Milenio ICM P02-054F, P.A.O. also thanks FONDECYT (grants 1020269 and 7020269), and J.M.Y. and G.A.L. thank U.A. (PEI-1305-04). E.V Anda acknowledges support from the brazilian agencies $\mathrm{CNPq}$ (CIAM project) and FAPERJ.

[1] L. I. Glazman, M. É. Raikh, JETP Lett. 47 (8) (1988) 452-455; D. Goldhaber-Gordon, H. Shtrikman, D. Mahalu, D. AbuschMagder, U. Meirav, and M. A. Kastner, Nature 391 (6663), 156 (1998); D. Goldhaber-Gordon, J. Göres, M. A. Kastner, H. Shtrikman, D. Mahalu, and U. Meirav, Phys. Rev. Lett. 81 (23), 5225 (1998); S. M. Cronenwett, T. H. Oosterkamp, and L. P. Kouwenhoven, Science 281 (5376), 540 (1998).

T. K. Ng, P. A. Lee, Phys. Rev. Lett. 61 (15) (1988) 1768-1771.

[2] A. Georges, Y. Meir, Phys. Rev. Lett. 82 (17), 3508 (1999); T. Aono, M. Eto, Phys. Rev. B 63 (12), 125327 (2001); H. Jeong, A. M. Chang, and M. R. Melloch, Science 293 (5538), 2221 (2001); Q. Feng Sun, H. Guo, Phys. Rev. B 66 (15), 155308 (2002).

[3] G. A. Lara, P. A. Orellana, and E. V. Anda, Solid State Comm. 125 (3-4), 165 (2003).

[4] P. Debray, O. E. Raichev, P. Vasilopoulos, M. Rahman, R. Perrin, and W. C. Mitchell, Phys. Rev. B61 (16), 10950 (2000).

[5] K. Kang, S. Y. Cho, J.-J. Kim, S.-C. Shin, Phys. Rev. B 63 (11), 113304 (2001); M. E. Torio, K. Hallberg, A. H. Ceccatto, and C. R. Proetto, Phys. Rev. B 65 (8), 085302 (2002); P. A. Orel- lana, F. Domínguez-Adame, I. Gómez, and M. L. L. de Guevara, Phys. Rev. B 67 (8), 085321 (2003); R. Franco, M. S. Figueira, and E. V. Anda, Phys. Rev. B 67 (15), 155301 (2003); P. Stefański, Solid State Comm. 128 (1), 29 (2003).

[6] J. Göres, D. Goldhaber-Gordon, S. Heemeyer, and M. A. Kastner, Phys. Rev. B 62 (3), 2188 (2000); I. G. Zacharia, D. Goldhaber-Gordon, G. Granger, M. A. Kastner, Y. B. Khavin, H. Shtrikman, D. Mahalu, and U. Meirav, Phys. Rev. B64 (15), 155311 (2001).

[7] U. Fano, Phys. Rev. 124 (6), 1866 (1961).

[8] E. Tekman, P. F. Bagwell, Phys. Rev. B 48 (4), 2553 (1993).

[9] J. U. Nöckel, A. D. Stone, Phys. Rev. B50 (23), 17415 (1994).

[10] G. Kotliar, A. E. Ruckenstein, Phys. Rev. Lett. 57 (11), 1362 (1986), and references cited therein.

[11] B. Dong, X. L. Lei, Phys. Rev. B63 (23), 235306 (2001); B. Dong, X. L. Lei, J. Phys.: Condens. Matter 13 (41), 9245 (2001); B. Dong, X. L. Lei, Phys. Rev. B 65 (24), 241304(R) (2002); B. Dong, X. L. Lei, Phys. Rev. B 66 (11), 113310 (2002).

[12] P. Coleman Phys. Rev. B 29 (6), 3035 (1984). 